\title{
Partikelmessungen, Abgasgrenzwerte, Stickoxide, Toxikologie und Umweltzonen
}

\author{
Eckard Helmers
}

Erhalten: 30. August 2008/Akzeptiert: 20. September 2008/Online veröffentlicht: 22. Oktober 2008

(C) Springer-Verlag 2008

\section{Einleitung}

Die internationale Konferenz befasst sich schwerpunktmäßig mit Partikelemissionen von Verbrennungskraftmaschinen, darüber hinaus aber mit dem Gesamtkomplex der Emissionen, ihrer gesundheitlichen Wirkung, möglichen Gegenmaßnahmen und dem legislativen Umfeld. Im Folgenden werden die in Zürich präsentierten Ergebnisse im Hinblick auf Ihre Umwelt- und Gesundheitsrelevanz aufbereitet, in Zusammenhänge eingeordnet und zum Teil kommentiert.

Zunächst geht es darum, wann und welche Emissionsdaten von Fahrzeugen überhaupt eine reale Basis für die Bewertung ihrer Umwelt- und Gesundheitswirkungen darstellen und mit welchen Einschränkungen diese zu handhaben sind. Die vielfältigen Probleme hierbei sind außerhalb der Fachwelt wohl weitgehend unbekannt.

\section{Validität von KFZ-Partikelmessungen auf Prüfständen}

Die Messtechnik ist traditionell und mit Recht ein Schwerpunkt der Tagung. Aus den aktuellen Beiträgen dieses

\section{Glossar:}

$\mathrm{PM}=$ partikuläres Material (Feinstaub)

PM10 = PM um $10 \mu \mathrm{m}$ Teilchengröße

$\mathrm{DPF}=$ Dieselpartikelfilter

$\mathrm{DOC}=$ Dieseloxidationskatalysator

$\mathrm{SCR}=$ selektive katalytische Reaktion (zur $\mathrm{NO}_{\mathrm{x}}$-Minderung)

$\mathrm{VOC}=$ flüchtige organische Kohlenstoffverbindungen

$\mathrm{PAH}=$ polyzyklische aromatische Kohlenwasserstoffe

\section{E. Helmers $(\bowtie)$}

Fachhochschule Trier, Umweltcampus Birkenfeld, Postfach 1380,

55761 Birkenfeld, Deutschland

E-Mail: e.helmers@umwelt-campus.de
Jahres wurde exemplarisch deutlich, wie schwierig es ist, valide Partikeldaten zu erhalten. Die Vortragenden aus verschiedenen Ländern präsentierten unterschiedliche, z. T. widersprüchliche Zahlen. Es erscheint deshalb geboten, einige Gründe für mangelnde Vergleichbarkeit und ggf. Validität solcher Daten zusammenzufassen:

1. Eine Reihe von Faktoren beeinflusst bereits die Messungen auf Motorprüfständen (I. Khalek, San Antonio, USA). Hier sind Drehzahl, Drehmoment und Leistung der Motoren zu nennen. Die Höhe der Messungen über Normalnull, die Umgebungstemperatur sowie die Temperatur des vor den Detektoren abgekühlten Gases wirken sich ebenfalls die Partikelzusammensetzung aus. Einen starken Einfluss auf die Partikelbildung hat die Mitverbrennung von Motoröl, wie sie bei älteren Autos häufig vorkommt. Entscheidend beeinflusst die Zusammensetzung des Treibstoffs und hierbei insbesondere der Schwefelgehalt die Messungen. Konzentration, Durchmesser und Volumen von Partikeln im ,Nucleation mode" sind mit dem Schwefelgehalt korreliert, sie entstehen aus Vorläufergasen wie $\mathrm{H}_{2} \mathrm{SO}_{4}$ (F. Arnold, Mainz). In diesem Zusammenhang ist von Bedeutung, dass Motorprüfstandsmessungen häufig mit speziellem, im Alltag nicht verfügbaren Prüfdiesel durchgeführt werden.

2. Es gibt verschiedene Methoden der Partikelanalyse. Deren Detektoren weisen unterschiedliche Empfindlichkeit für verschiedene Partikelgrößen auf (B. Gorbunov, Univ. of Kent, GB). Deswegen sind sie vor der Messung mit einem speziellen Kalibrationsaerosol einzustellen, welches den realen Proben möglichst ähnlich sein sollte (T. Yamashita, Toyota Motor Corp., Japan).

3. Die fast ausschließlich auf Motorprüfständen gemessenen Daten lassen sich nicht ohne weiteres auf tatsächliche Verkehrsemissionen übertragen. Dies gilt besonders 
für Emissionen von Dieselmotoren, die in Dauertests ansteigende Tendenz zeigten (dokumentiert in der Motorpresse). Schon bei dem Problem der Edelmetallemissionen aus Abgaskatalysatoren zeigte sich, dass Motoren mit Defekten wie Fehlzündungen um Größenordnungen höhere Emissionen hatten, die auch nach Behebung eines Schadens deutlich angehoben blieben (Helmers 1999). Verschiedene Arbeitsgruppen haben deshalb sehr aufwändige on-road-Messlabors realisiert, z. B. untergebracht in US-amerikanischen Trucks, deren Abgase unter Straßenbedingungen innerhalb des mobilen Labors verarbeitet und analysiert werden. H. Jung (Univ. of California) fand in solchen Messungen überraschend hohe Partikelemissionen eines großen LKW und schlussfolgerte, dass die ,normierten Tests nicht ausreichen, um den Partikelausstoß unter Straßenbedingungen zu verstehen oder zu charakterisieren."

4. Bekanntlich wird besonders in Deutschland anders gefahren, als es die normierte Simulation auf Prüfständen wiedergibt. Der Neue Europäische Fahrzyklus von 1996 und der EC-2000-Zyklus sehen Geschwindigkeiten von $\max .120 \mathrm{~km} / \mathrm{h}$ vor. Oberhalb dieser Geschwindigkeit steigen die Emissionen deutlich an. Die Diskrepanz zwischen normierten Schadstoffemissionen und Realität wurde in Zürich von L.M. Vijtisek (Univ. von Liberec) für Tschechien angesprochen in seinem Beitrag: „Growth in road traffic, off-cycle emissions, aggressive driving and real-world PM emissions: Can the benefits of new vehicles keep up?" Vijtisek sieht einen Zusam- menhang zwischen dem Anstieg des Dieselverbrauchs und dem Anstieg verschiedener Atemwegserkrankungen in den letzten Jahren in Tschechien. Er erkennt insbesondere in der realen, fluktuierenden Verkehrssituation eine Ursache für hohe Emissionen. Der tatsächliche Partikelund $\mathrm{NO}_{\mathrm{x}}$-Ausstoß kann nach den von Vijtisek präsentierten Zahlen für moderate Geschwindigkeiten bis zu vierfach über den EU-Grenzwerten liegen.

\section{Zukünftige EU-Abgasgrenzwerte für PKW}

Ein Thema auf vielen Ebenen ist die zukünftige EU-Abgasgesetzgebung, die in enger Abstimmung mit den Fahrzeugherstellern weiterentwickelt wird (Tabelle 1). In diesem Zusammenhang wurden EU-5- und EU-6-Grenzwerte auf der Tagung mehrfach angesprochen. Auf dieser Ebene sollen erstmals die Partikelzahlen begrenzt werden, wobei aus messtechnischen Gründen Partikel erst ab einer Größe von $23 \mathrm{~nm}$ berücksichtig werden (H. Jung, Univ. of California). In diesem Zusammenhang sei erwähnt, dass die aus Gasen entstehenden sekundären Partikel Größen von $1 \mathrm{~nm}$ aufwärts aufweisen (nucleation mode), worauf sie zu größeren Partikeln anwachsen. Die bei der Verbrennung außerdem gebildeten primären Rußpartikel haben eine Größe ab 40-100 nm (F. Arnold, Mainz).

Eine aktuelle Übersicht über den Stand der drei führenden Normensysteme auf der Welt (siehe Tabelle 1) lässt folgende Entwicklungen erkennen:

Tabelle 1 Aktueller Planungsstand der EU-Emissionsgrenzwerte für PKW im Vergleich zur Gesetzgebung in den USA und Japan. Quellen: T. Yamashita, Toyota Motor Corp., UBA (2003), Dieselnet (2008)

\begin{tabular}{|c|c|c|c|c|c|c|}
\hline \multirow[t]{2}{*}{ Grenzwert } & \multicolumn{2}{|c|}{ Partikelzahl $\left(\mathrm{km}^{-1}\right)$} & \multicolumn{2}{|c|}{ Partikelmasse $\left(\mathrm{mg} \mathrm{km}^{-1}\right)$} & \multicolumn{2}{|c|}{ Stickoxide (mg km ${ }^{-1}$ ) } \\
\hline & Diesel & Benziner $^{1}$ & Diesel & Benziner $^{1}$ & Diesel & Benziner \\
\hline EU 4 & - & - & 25 & - & 250 & 80 \\
\hline $\begin{array}{l}\text { EU 5a } \\
\text { ab } 2009\end{array}$ & - & - & 5 & 5 & 180 & 60 \\
\hline $\begin{array}{l}\text { EU 5b } \\
\text { um } 2011\end{array}$ & $6 \times 10^{11}$ & - & $3-4,5$ & $3-4,5$ & 180 & 60 \\
\hline $\begin{array}{l}\text { EU } 6 \\
\text { um } 2014\end{array}$ & $6 \times 10^{11}$ & Noch nicht entschieden & $3-4,5$ & $3-4,5$ & 80 & 60 \\
\hline $\begin{array}{l}\text { TIER } 2 \\
\text { USA, } 2009\end{array}$ & - & - & 16 & 16 & $110^{2)}$ & $110^{2)}$ \\
\hline $\begin{array}{l}\text { ULEV }^{3} \\
\text { USA, } 2004\end{array}$ & - & - & 16 & 16 & 78,5 & 78,5 \\
\hline $\begin{array}{l}\text { SULEV }^{3} \\
\text { USA, bis } 2010\end{array}$ & - & - & 16 & 16 & 31 & 31 \\
\hline Japan 2009 & - & - & 5 & 5 & 80 & 50 \\
\hline Japan $>2009$ & - & - & & EV: weitere & uktion an & \\
\hline
\end{tabular}

1) Nur Direkteinspritzer-Benziner.

2) Bis 4,5 t zul. Gesamtgewicht (auch Nutzfahrzeuge und Busse, Mittelwert). Hinweis: Die US-amerikanischen Abgasnormen sind komplexer gefasst und auch schwerer einzuhalten; beispielsweise müssen die Emissionsgrenzen bis zu 150.000 Meilen bzw. mind. 10 Jahre garantiert werden. Der Fahrzyklus unterscheidet sich und generiert höhere $\mathrm{NO}_{\mathrm{x}}$-Emissionen als der europäische.

3) Werte für PKW. SULEV in vier US-Bundesstaaten bereits in Kraft gesetzt. 
1. Die EU-Kommission setzt mit den neuen Grenzwerten Euro-5 und Euro-6 ihre Tradition fort, lediglich den bereits erreichten technischen Ist-Zustand mehrere Jahre in der Zukunft festzuschreiben. Anregungen, neue Techniken zu entwickeln oder Emissionen signifikant zu reduzieren, gehen von den EU-Grenzwerten für PKW mindestens bis 2014 nicht aus, außer derzeit in begrenztem Rahmen für $\mathrm{CO}_{2}$ (hier nicht gezeigt). Der EU-5-Standard für Partikelmasse kann von den bereits jetzt angebotenen Fahrzeugen mit Partikelfilter erreicht werden. Deren Technik führt derzeit jedoch zu einem erheblichen Anstieg von $\mathrm{NO}_{2}$.

2. Die EU-Kommission erlaubt Diesel-PKW bis 2014 deutlich höhere Stickoxid-Emissionen als benzinbetriebenen PKW. Dies ist unter den drei führenden Normen einzigartig. Erst ab frühestens 2014 sollen in der EU die Diesel$\mathrm{NO}_{\mathrm{x}}$-Emissionen auf das Niveau des Benziners gesenkt werden, was bereits heute in die USA exportierte Fahrzeuge mit Harnstoff-Einspritzung erreichen. Dies sichert der EU-Autoindustrie im heimischen Bereich den Absatz der Dieselfahrzeuge bis 2014, da die $\mathrm{NO}_{\mathrm{x}}$-Reduktion auf das in den USA verlangte Niveau die Autos signifikant verteuert. Diese Politik ist umso erstaunlicher angesichts der aktuellen hohen Stickoxidbelastung in europäischen Großstädten und weil bereits absehbar ist, dass dort der ab 2012 geltende EU-Grenzwert für $\mathrm{NO}_{2}$ von $30 \mu \mathrm{g} \mathrm{m}^{-3}$ nicht eingehalten werden kann (siehe unten: Umweltzonen).

3. Ein Fortschritt ist die Einbeziehung der Partikelzahlen in EU-5b, da dies die toxikologisch wichtigere Komponente ist. Abgastechnisch ergibt sich hieraus keine Herausforderung, da nach T. Yamashita Partikelmassen und -zahlen stark korreliert sind.

4. Die drei führenden Emissionsstandards haben einen enormen Stellenwert, weil sie weltweit übernommen werden. So wurden die EU-Emissionsnormen von Indien und China adaptiert. Selbstverständlich spielt auch die Normengebung für LKW-Emissionen eine wichtige Rolle. Doch konkurrieren hier nicht verschiedene Motortechniken wie bei den PKW.

Im Bericht über die 10. ETH-Conference on Combustion Generated Nanoparticles (Helmers 2006) wurde bemängelt, dass kaum Daten zur Verfügung stehen, die einen direkten Vergleich der Emissionen verschiedener Motortechniken erlauben. Auf der diesjährigen Tagung präsentierte T. Yamashita jedoch eine Übersicht der Partikelemissionen (Teilchenzahlen) verschiedener PKW der Firma Toyota, die einen solchen Technikvergleich ermöglicht (Abb. 1). Hieraus ergibt sich:

1. (nur) mit Partikelfilter erreichen Diesel-PKW die niedrigen Partikelemissionen von benzinbetriebenen PKW mit (klassischer) Multipoint-Einspritzung. Ohne Partikelfilter liegen die Partikelemissionen von Diesel-PKW 500 bis 1.000 fach höher.

2. Direkteinspritzer-Benziner ohne Partikelfilter emittieren zehn- bis hundertfach mehr Partikel als PKW mit her-
Partikel-

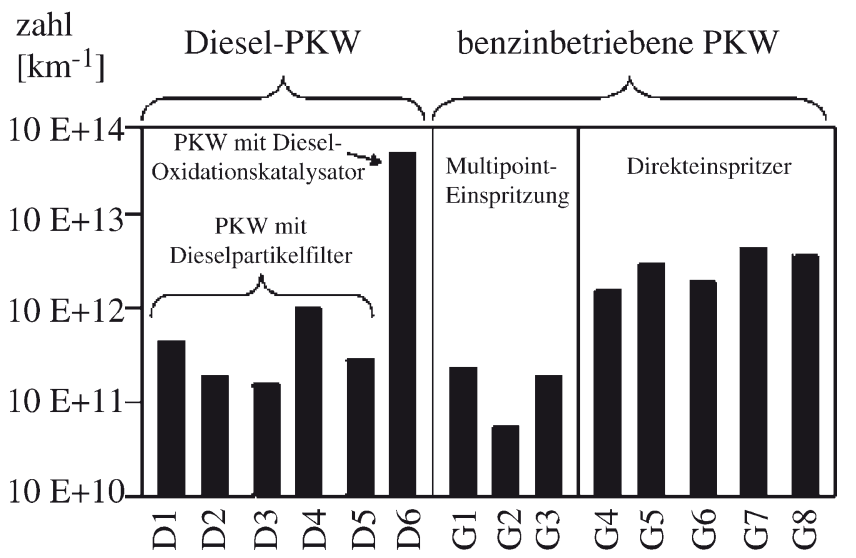

Abb. 1 Partikelemissionen von Toyota-PKW. Partikelzahlen: logarithmische Auftragung. Nach T. Yamashita, Toyota Motor Corporation Erläuterungen:

D1-D6 = 4-Zyl.-Direkteinspritzer-Diesel, Hubraum 2-2,2 1, EU-4-

klassifiziert

D1-D3 = Fahrzeuge mit DPNR (Diesel particulate $\mathrm{NO}_{\mathrm{x}}-$ Reduction catalyst)

D4 + D5 = Fahrzeuge mit DPF (Dieselpartikelfilter)

D6 = Fahrzeug mit Dieseloxidationskatalysator (DOC) zur Partikel-

reduktion

G1-G8 = Benzinbetriebene PKW mit Dreiwege-Katalysator

$\mathrm{G} 1-\mathrm{G} 3=6-$ Zylinder-Multipointeinspritzer mit 31 Hubraum

G4-G8 = V6-Direkteinspritzer (DI) mit 2,5-4,6 1 Hubraum

kömmlicher Benzineinspritzung und fünf- bis fünfzigmal mehr Partikel als die betrachteten Diesel-PKW mit Partikelfilter. Hierbei ist allerdings zu berücksichtigen, dass die Hubräume der betrachteten DirekteinspritzerBenziner etwa doppelt so groß sind.

3. Hieraus ergibt sich tatsächlich die Notwendigkeit, die Partikelemissionen von Benzin-Direkteinspritzern zu überwachen. Die Emissionen kleiner Direkteinspritzer von etwa 1,4 Litern Hubraum, wie sie in Deutschland produziert werden, dürften jedoch niedriger liegen.

4. Vor dem Hintergrund der etwa fünffach niedrigeren Stickoxidemissionen ergeben sich für benzinbetriebene PKW immer noch erhebliche Umweltvorteile trotz Partikelfilterung bei Dieseln.

Die Größenverteilung der emittierten Partikel unterscheidet sich nach T. Yamashita: Ein Diesel-PKW mit DPNR weist ein Maximum der Größenverteilung bei $45 \mathrm{~nm}$ auf, die damit verglichenen 3-1-DI-Benzinmotoren ein Maximum bei $80 \mathrm{~nm}$ Teilchengröße.

\section{Weitere Quellen von Partikelemissionen}

Auch die Emissionen von Düsenflugzeugen haben Auswirkungen auf die Umweltqualität, insbesondere in der Nähe von Flughäfen. Entsprechend berichtete D. Hagen (Rolla, 
Missouri, USA) über die Partikelgrößenverteilung von Passagierjets.

Zunehmend und besonders in der Schweiz stellen Holzheizungen einen großen oder dominierenden Anteil der Partikelemissionen. M. Sattler (Langenbruck, CH) stellte Messmethoden zur elementar- und Partikelcharakterisierung von Holzheizungsabgasen vor. Die Erkenntnisse sind unverzichtbar zur Optimierung solcher Öfen. A. Richard (Villigen, $\mathrm{CH}$ ) untersuchte die Emissionen von Holzpelletöfen.

\section{Partikeldynamik, sekundäre Emissionen von Partikelfiltern, Toxikologie und Gesundheitswirkungen}

Die geplante Einbeziehung der Partikelzahlen in die EUAbgasgesetzgebung (siehe Tabelle 1) folgt dem toxikologischen Erkenntnisgang der letzten Jahre. Allerdings scheint die toxikologische Bewertung bereits einen Schritt weiter zu sein. Wie E. Karg vom Münchener Helmholtz-Zentrum ausführte, zeigten wegweisende Veröffentlichungen von Stoeger und Oberdörster, dass die Entzündungsantwort nach der Exposition gegenüber synthetischen Partikeln fast doppelt so gut mit der Oberfläche korreliert wie mit der Partikelzahl. Das war die Motivation für Karg et al., ein Verfahren für die Bestimmung von Partikeloberflächen zu entwickeln.

Für die toxikologische Wirkung von Dieselruß ist wesentlich die adsorbierte Schicht von Kohlenwasserstoffen verantwortlich. Hierbei handelt es sich großenteils um polyzyklische aromatische Kohlenwasserstoffe (PAH). Der Bildungsmechanismus dieser komplexen Stoffgruppe wurde von M. Kraft (Cambridge) diskutiert.

A. Peters (München) fasste die bisher verfügbaren epidemiologischen Studien zur Wirkung von Feinstaub zusammen. Verkehrsemissionen sind danach für zusätzliche Sterblichkeit als Folge von Herzkreislauferkrankungen und Arteriosklerose verantwortlich. Sie sind darüber hinaus beteiligt an der Entwicklung und Ausprägung von Allergien. Positive Effekte sind deshalb nach Einrichtung von Umweltzonen zu erwarten, die größer ausfallen dürften als aufgrund des Rückgangs an PM10 zu erwarten. In der anschließenden Diskussion ging es um die oft gestellte Frage, ob die genannten gesundheitlichen Effekte nicht auch auf den Verkehrslärm zurückzuführen sein können. A. Peters merkte dazu an, dass Lärm zwar ebenso zu Herz-KreislaufErkrankungen führe, die spezifischen Entzündungseffekte von Partikeln aber davon unterschieden werden könnten. In anderen Worten: Pathomechanistisch agieren Partikel und Lärm unabhängig von einander.

Oravisjäri (Oulu) stellt Untersuchungen zur Größenklassenfraktionierung des Depositionsverhaltens von Feinstaub in den menschlichen Atemwegen vor. Die Daten berück- sichtigen den Einfluss des Geschlechts und der körperlichen Aktivität.

L. Müller (Bern) bestimmte die Toxizität der Emissionen von Motorrollern in Versuchen mit menschlichen Bronchialepithelzellen. Hierbei wurde der oxidative Stress anhand von DNA-Beschädigungen nachgewiesen. Ein positiver Effekt der Abgasnachbehandlung zeigte sich bei den oxidativen Beschädigungen und bei Entzündungseffekten, nicht aber bei der Zytotoxizität. Interessanterweise führt auch die gegenwärtig bei manchen Motorrollern verfügbare Abgasbehandlung zu einer Zunahme der Partikel im toxikologisch besonders interessanten Größenbereich von $10-100 \mathrm{~nm}$.

A. Baeza (Paris) fasste ein aufwendiges Projekt zusammen, in dem die proentzündliche Wirkung von in Paris gesammeltem Staub in Atemwegen quantifiziert wurde. Auch die chemische Zusammensetzung von vier Größenfraktionen des Pariser Feinstaubs zwischen 0,03 und $10 \mu \mathrm{m}$ wurde dabei bestimmt. Volumennormiert hatte die Partikelfraktion von 0,1 bis $1 \mu \mathrm{m}$ die größte Wirkung auf exponierte Zellen, massennormiert die Fraktionen von 0,03 bis $1 \mu \mathrm{m}$. A. Baeza schlussfolgerte, dass zumindest in Paris die PM1-Fraktion die städtische Belastungssituation besser beschreibt als die PM10-Feinstaubfraktion.

C. Sioutas (Los Angeles) informierte über die Erforschung der physikochemischen und toxikologischen Eigenschaften der Partikelemissionen von schweren LKW mit und ohne fortschrittlicher Abgastechnologie. Die Untersuchung von sieben verschiedenen Abgasreinigungstechniken ergab folgendes klares Ergebnis: Die Minderung der Partikelmasse geht mit der Erhöhung der Partikelzahl einher. In Zahlen: Die Senkung der Partikelmasse von etwa 100 auf etwa $2 \mathrm{mg} \mathrm{km}^{-1}$ korreliert gleichzeitig mit einem Anstieg der Partikelzahlen von $2 \times 10^{14}$ auf $7 \times 10^{15}$ Partikel km${ }^{-1}$. Der stärkste Effekt wurde mit einem SCRT-System erzielt (selective catalytic reaction und Partikelfilter). Damit wird die physikochemische Grundannahme bestätigt, dass bei Entfernung der Feststofffraktion durch einen Partikelfilter die Zahl der kleineren Partikel zunimmt, da diese sich nicht mehr an die Feststoffteilchen anlagern können. In Toxizitätstests zeigte sich jedoch eine deutliche Abnahme der Redoxaktivität von Partikeln aus LKW mit moderner Abgastechnologie. Dies galt umgerechnet auf den Fahrzeugkilometer, jedoch nicht bezogen auf die Partikelmasse.

Auch M. Gautam (Morgantown, West Virginia, USA) bestätigte die Zunahme der Nanopartikel durch Abgasnachbehandlung bei schweren LKW.

Ebenso zeigten die Untersuchungen von A. Virtanen (Tampere), dass sich die Größenverteilung sowie (als Ursache dafür) die Partikelchemie durch unterschiedliche Abgasnachbehandlungen grundlegend wandelt. Virtanen et al. quantifizierten den Einfluss von Oxidationskatalysatoren und Partikelfiltern bei Diesel-PKW. 
R. Vogt (Ford Deutschland) stellte Messungen vor, welche die Partikelemissionen während der Regenerationszeiten eines Dieselpartikelfilters (DPF) erhellen. Die Zahl der emittierten Nanopartikel steigt während dieser Phasen um das $10^{3}$ bis $10^{4}$ fache an. Sie sind offenbar mit im DPF gespeicherten Schwefelverbindungen korreliert.

Die sekundären Effekte von DPF waren einmal mehr Thema von N. Heeb (Dübendorf, CH). Fokus seiner Forschungen ist das Verhalten von DPF im Hinblick auf Dutzende karzinogener, mutagener und hormonähnlicher Komponenten im Dieselabgas. PAHs werden nach diesen Untersuchungen effizient zurückgehalten. Auf der anderen Seite kann ein DPF als chemischer Reaktor gentoxische Nitro-PAHs erzeugen. Dies ist wie die bereits früher veröffentlichte Dioxinproduktion von DPF jedoch stark vom Filtermaterial abhängig. Die schweizerische VERT-Liste empfielt nur DPF, die auf solche sekundären Emissionen geprüft sind.

Ein verbreiteter „sekundärer Effekt“ von DPF ist die zum Teil stark erhöhte $\mathrm{NO}_{2}$-Emission. Diese bestätigt $\mathrm{S}$. Niemi (Vasa, Finnland) für vier verschiedene untersuchte Filtertypen bei sehr unterschiedlicher Filtereffizienz. Der Anteil von $\mathrm{NO}_{2}$ betrug maximal $58 \%$ des gesamten emittierten $\mathrm{NO}_{\mathrm{x}}$. Nur einer von vier verschiedenen DPF zeigte akzeptable $\mathrm{NO}_{2}$-Emissionen. Interessant war die Untersuchung eines modernen SCR-Systems zur Minimierung der $\mathrm{NO}_{\mathrm{x}}$ Emissionen. Dieses filterte lediglich die großen Partikel effizient, jedoch die kleinen Nanopartikel kaum aus dem Abgas heraus. S. Niemi wies ebenso darauf hin, dass die Filtertemperaturen bei der praktischen Anwendung für eine passive Reinigung zu niedrig seien.

Das quantitative Ausmaß der zusätzlichen $\mathrm{NO}_{2}$-Emissionen durch DPF zeigten Mayer et al. in einem Poster über Euro-3-, Euro-4- und Euro-5-Schwerlastfahrzeuge mit und ohne DPF: $\mathrm{NO}_{2}$ steigt nach diesen Daten im Abgas von ca. $10 \mathrm{ppm}$ vor einem DPF auf bis zu $350 \mathrm{ppm}$ nach DPF an. Außerdem wurde der Abbau von NO durch einen SCR-Kat gemessen: NO sinkt dabei von 1150 auf 100-300 ppm ab. In der Euro-4-Version sind bei LKW wie bei Diesel-PKW hohe $\mathrm{NO}_{2}$-Konzentrationen für die Regeneration des PM-Kats erforderlich. Dennoch sei die Partikelemission beim Euro-4PM-Kat unvorhersagbar. Euro-5-Denox-Systeme würden nur noch vernachlässigbare $\mathrm{NO}_{2}$-Gehalte emittieren.

Bei $\mathrm{NO}_{2}$-Emissionen um 350 ppm wird auch klar, dass dieses Gas von Verkehrsteilnehmern direkt wahrgenommen werden kann: Die Geruchsschwelle von $\mathrm{NO}_{2}$ liegt bei 0,1 bis $0,5 \mathrm{ppm}$.

Die hohen $\mathrm{NO}_{2}$-Emissionen von Euro-4-PKW und -LKW werfen die Frage auf, ob der berechnete epidemiologische Nutzen (Gesundheitsvorteil der Bevölkerung, siehe: Wichmann 2004) im Hinblick auf die flächendeckende Einführung von DPF in der erwarteten Weise eintreffen wird: Gesundheitsbeeinträchtigungen durch die zunehmende Exposition gegenüber $\mathrm{NO}_{2}$ dürften hierbei nicht eingerechnet sein.

\section{Einrichtung von Umweltzonen in europäischen Großstädten}

Im anschließenden Fokusevent ging es um Verkehrsleitmaßnahmen zur Emissionsreduktion in Europa: die Einrichtung von Umweltzonen. Deren Einrichtung ist folgerichtig, denn die tatsächlichen Verkehrsemissionen sind in europäischen Großstädten trotz aller Abgasminderungsmaßnahmen aktuell zu hoch. Dies gilt besonders für stagnierende oder ansteigende Ozonwerte.

M. Lutz (Berlin) berichtet über die Planung der Umweltzone in den inneren Bezirken Berlins. Eine Bilanzierung hatte ergeben, dass mehr als die Hälfte der Partikelimmission (PM10) städtischen Quellen entstammt. Weil auch hier Dieselfahrzeuge als Ursache angesehen werden, sollen Fahrverbote primär ältere Diesel-PKW sowie Benzin-KFZ ohne Abgaskatalysator betreffen. Die daraus für das Jahr 2010 erwarteten Emissionsrückgänge sind erheblich:

- $10 \%$ für $\mathrm{NO}_{\mathrm{x}}$,

- $45 \%$ für Feinstaub aus Abgasen,

- $9 \%$ für Feinstaub allgemein.

Weitere Verkehrsleitmaßnahmen sollen diese Werte anheben auf

- $22 \%\left(\mathrm{NO}_{\mathrm{x}}\right)$,

- $53 \%$ (Feinstaub aus Abgasen) bzw.

- $21 \%$ (Feinstaub allgemein).

Eine Grafik der PM10-Belastungsstruktur innerhalb Berlins (Abb. 2) zeigt die hohen Belastungsspitzen an Hauptstraßen sowie die dafür verantwortlichen Quellen. Aus diesem Sachverhalt (siehe Abb. 2) ergibt sich schnell der Sinn und Nutzen von Verkehrsbeschränkungen. Ursächlich für die Einrichtung der deutschen Umweltzonen ist der seit 1. Januar 2005 geltende EU-Grenzwert von $40 \mu \mathrm{g}$ PM10 $\mathrm{m}^{-3}$. Als Problem sieht M. Lutz die hohen Stickoxidund besonders $\mathrm{NO}_{2}$-Emissionen der Diesel-PKW, die auch mit dem neuen EU-5-Standard nicht signifikant sinken werden (siehe Tabelle 1). Für die Bilanzierung der Emissi-

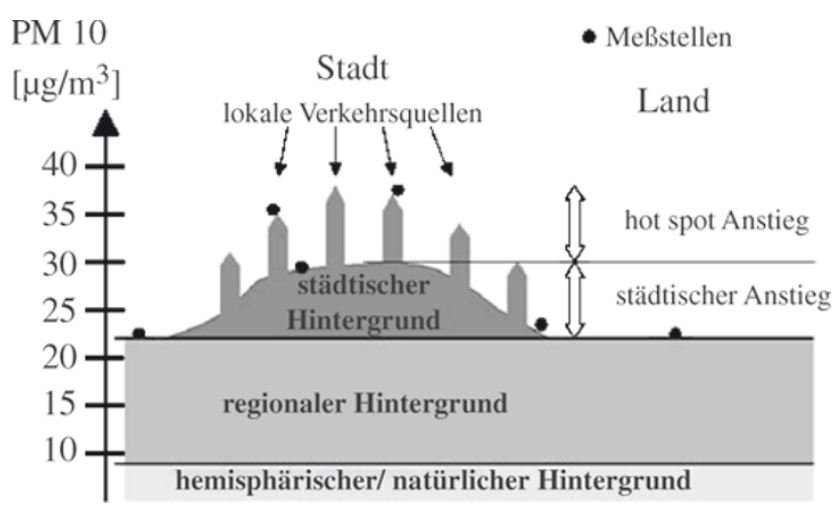

Abb. 2 Belastung einer Großstadt mit Feinstaub der Kategorie PM10 am Beispiel Berlins (Jahresmittelwerte, nach M. Lutz, Berlin) 
onen von EU-4-Diesel-PKW wurden in Berlin $200 \mathrm{mg}$ NO sowie $150 \mathrm{mg} \mathrm{NO} \mathrm{km}^{-1}$ angenommen. Bei EU-4-Dieseln mit Partikelfilter wurden $160 \mathrm{mg} \mathrm{NO}$ und $200 \mathrm{mg} \mathrm{NO}_{2} \mathrm{~km}^{-1}$ zugrunde gelegt. Bei EU-5-Diesel-PKW geht man in Berlin von $150 \mathrm{mg}$ NO sowie $100 \mathrm{mg} \mathrm{NO}_{2} \mathrm{~km}^{-1}$ aus (M. Lutz). Die Werte sind realistisch oberhalb der Emissionsgrenzwerte (siehe Tabelle 1) angesetzt, da diese in Europa nicht über die Lebenszeit eines Fahrzeugs hinweg eingehalten werden müssen.

Probleme mit der Einhaltung der EU-Grenzwerte für PM10 und $\mathrm{NO}_{2}$ stellten auch die Motivation für die Einrichtung der Umweltzone von Stockholm im Jahr 2006 (C. Johansson, Stockholm). Die Konzentrationen beider Schadstoffe sind in Stockholm eng miteinander korreliert.

Im Jahr 2006 gingen dann in Stockholm die Schadstoffkonzentrationen von $\mathrm{NO}_{\mathrm{x}}$ um 8,5\% und die von CO, PM10, VOC sowie Benzol um 12-14\% im Vergleich zur Situation vor Einrichtung der Umweltzone zurück. Auf der Basis von epidemiologischen Mortalitätsraten konnte der gesundheitliche Nutzen durch den Rückgang von PM10 und $\mathrm{NO}_{x}$ berechnet werden. Johansson gab an, dass der gemessene Rückgang von $0,23 \mu \mathrm{g} \mathrm{NO}_{\mathrm{x}} \mathrm{m}^{-3}$ bei einer Bevölkerung von 1,44 Mio. ca. 26 vorzeitige Todesfälle pro Jahr vermeidet. Er schlussfolgerte, dass die Schadstoffkonzentrationen weniger gesunken seien als erwartet, der Nutzen für die Bevölkerung sei jedoch hoch.

Die Umweltzone (low emission zone, LEZ) von London startete im Februar 2008 wegen erheblicher langjähriger Überschreitungen der PM10- und $\mathrm{NO}_{2}$-Standards. Als Hauptproblem werden auch hier Dieselfahrzeuge (LKW und PKW) angesehen, wobei Diesel-PKW den größten Emissionsanteil stellen.

Mittelfristig werden auch für London positive Gesundheitseffekte bei der Bevölkerung und damit auch erhebliche ökonomische Vorteile in dreistelliger Millionenhöhe erwartet (S. Beevers, London). Die PM10-Belastung stellt sich ähnlich dar wie in Berlin (siehe Abb. 2). Analog dazu kann auch die Stickoxidbelastung in London beschrieben werden: Einer Grundbelastung von knapp 50 ppb $\mathrm{NO}_{\mathrm{x}}$ stehen an verkehrsreichen Standorten Spitzenwerte von bis zu 240 ppb gegenüber. Die langjährigen Trends von $\mathrm{SO}_{2}$ und $\mathrm{CO}$ weisen in London nach unten, während PM10 und $\mathrm{NO}_{2}$ stagnieren und Ozon ansteigt. Darüber hinaus referierte Beevers Untersuchungen des oxidativen Potenzials von PM. Als Ursachen für dieses Potenzial wurden Metalle und Quinone identifiziert.

\section{Fazit}

Eine Gesamtsicht der Nachhaltigkeit von Mobilitätsansätzen fehlte auf der Konferenz, war aber auch nicht ihr Thema. Deswegen sei an dieser Stelle darauf hingewiesen, dass Fahrzeuge mit Verbrennungsmotoren wohl immer problematisch für Umwelt und Gesundheit bleiben. Dies gilt insbesondere für Fahrzeuge mit Dieselmotoren, deren energetische Effizienz (noch) nicht mit Umwelteffizienz gepaart ist; insbesondere in der EU sowie in den Ländern, die EU-Standards übernehmen. Doch Alternativen existieren: Inzwischen wird deutlich, dass Elektromobilität auch bei hauptsächlich fossiler Stromerzeugung keine Nachteile bei den $\mathrm{CO}_{2}$-Emissionen mit sich bringt. Dagegen wären die unmittelbaren Vorteile immens: Zum Beispiel könnten $\mathrm{PM}$ - und $\mathrm{NO}_{\mathrm{x}}$-Belastungsspitzen in Städten mehr und mehr entfallen. Die immensen Umwelt- und Gesundheitskosten der Mobilität mittels Verbrennungsmotoren schienen bislang unausweichlich und wurden auch auf dieser Tagung in ihren Einzelheiten betrachtet. Sie sind mit Elektromobilität zum großen Teil vermeidbar. Allerdings wird die Reduktion von Dieselemissionen immer ein Thema bleiben: Es geht um Dieselgeneratoren und -Fahrzeuge im Untertagebetrieb, um Schiffsdiesel, um landwirtschaftliche Fahrzeuge und natürlich um LKW in jeder Größe. Noch jahrzehntelang werden Millionen Alt-PKW und -LKW mit Dieselmotoren auf den Straßen fahren. Hier bleibt das „Retrofitting“ ein großes Thema, die Nachrüstung von Abgasbehandlung (Mayer 2008). Die Ergebnisse der Züricher Tagung zeigen auch die neue Dringlichkeit, Abgasnachbehandlungssysteme anzubieten und flächendeckend nachzurüsten, die $\mathrm{NO}_{\mathrm{x}}-$ Emissionen vermindern.

\section{Literatur}

UBA (2003) http://www.umweltbundesamt.de/verkehr/techemissmm/ dieselfahrzeuge.htm. 28.8.2008

Dieselnet (2008) http://www.dieselnet.com/standards. 28. 8. 2008

Helmers E (1999) Platinum emission rate of automobiles with catalytic converters. Environ Sci Pollut Res 4(2):100-103

Helmers E (2006) 10th ETH Conference on Combustion Generated Nanoparticles. UWSF - Z Umweltchem Ökotox 18(4):280-282

Mayer A et al. (2008) Particle filter retrofit for all diesel engines. Expert-Verlag, Renningen

Wichmann E (2004) Positive gesundheitliche Auswirkungen des Einsatzes von Partikelfiltern bei Dieselfahrzeugen - Risikoabschätzung für die Mortalität in Deutschland. Umweltmed Forsch Praxis 9(2):85-99 